\title{
Berberine, a natural compound, suppresses Hedgehog signaling pathway activity and cancer growth
}

\author{
Juan Wang ${ }^{1}$, Yuanqiu Peng ${ }^{1 \dagger}$, Yuan Liu ${ }^{1 \dagger}$, Jun Yang ${ }^{1}$, Ning Ding ${ }^{2,3}$ and Wenfu Tan ${ }^{1 *}$
}

\begin{abstract}
Background: Berberine (BBR), a natural alkaloid compound, is used as a non-prescription drug in China for treating diarrhea and gastroenteritis. Many studies have revealed that BBR possesses anticancer effect. However, the molecular mechanisms underlying its anticancer action is far from being fully elucidated. This study is aimed to determine the effect of BBR on the hedgehog $(\mathrm{Hh})$ activity and the growth of cancers addiction to Hh activity.

Methods: The Hh activity was determined by dual luciferase assays and quantitative RT-PCR analyses. The growth inhibition of BBR on medulloblastoma which was obtained from ptch+/-;p53-/- mice was analyzed by 5-bromo-2deoxyuridine (Brdu) assays and by allografting the medulloblastoma into nude mice. The data were statistically analyzed by one-way analysis of variance (ANOVA), and multiple comparison between the groups was performed using Dunnett's method.

Results: In this study, we found that BBR significantly inhibited the Hh pathway activity. Meanwhile, we observed that BBR failed to affect the transcriptional factors activities provoked by tumor necrosis factor-a (TNF-a) and Prostaglandin E2 (PGE2), thus suggesting its unique property against Hh pathway activity. Further studies revealed that BBR inhibited the Hh pathway activity by potentially targeting the critical component Smoothened (Smo) and most likely shared the same binding site on Smo with cyclopamine, a classical Smo inhibitor. Finally, we demonstrated that BBR obviously suppressed the Hh-dependent medulloblastoma growth in vitro and in vivo.

Conclusion: Collectively, our study uncovered a novel molecular mechanism responsible for the anticancer action of BBR, thus opening the way for the usage of BBR for therapeutics of cancers addiction to aberrant Hh pathway activity.
\end{abstract}

\section{Background}

Hh signaling pathway is an evolutionarily conserved signaling axis of embryonic patterning and tissue homeostasis [1, 2]. Deregulated activity of the Hh signaling pathway has also been shown to be involved in the development of tumors which arise sporadically or in genetically predisposed individuals. In vertebrates, three $\mathrm{Hh}$ ligands (Sonic hedgehog, Shh; Indian hedgehog, Ihh; Desert hedgehog, Dhh) have been identified that bind to the 12-transmembrane cell surface receptor Patched1 (PTCH1). When not bound by Hh ligands, PTCH1

\footnotetext{
* Correspondence: wftan@fudan.edu.cn

${ }^{\dagger}$ Equal contributors

'Department of Pharmacology, School of Pharmacy, Fudan University, 826

Zhangheng Road, Shanghai 201203, P.R. China

Full list of author information is available at the end of the article
}

restrains the activity of Smo, a member of the 7transmemebrane cell surface receptor. However, upon bound by a ligand, PTCH1 no longer inhibits Smo, thus allowing the accumulation of Smo in the primary cilium. Ultimately, canonical Hh signaling regulates the activity, proteolytic processing, and the stability of the Gli family transcriptional factors, Gli1-3, and subsequently initiates the transcription of Gli-dependent target genes, such as Gli1 and ptch1 [3]. This regulation requires a number of protein kinases, including protein kinase A, glycogen synthase kinase 3 and casein kinase 1, and the negative regulator suppressor of fused $(\mathrm{SuFu})$ [4].

The mechanisms responsible for the constitutive $\mathrm{Hh}$ pathway activity in cancers include ligand-independent and ligand-dependent manner. Ligand-independent constitutive 
activation of Hh pathway in cancers is characterized by somatic mutations in Ptch1, Smo, or Sufu, consequently increasing Hh pathway activity and leading to a higher incidence of basal cellular carcinoma (BCC) and medulloblastoma. On the other hand, aberrant Hh pathway activity in a wide range of cancers is associated with elevated $\mathrm{Hh}$ ligands secretion from tumors or the stromal environment, namely ligand-dependent manner $[2,5]$. Large-scale efforts have been made to develop Hh pathway antagonists for treatment of cancers. As a central regulator of the pathway and a readily accessible cell surface component, Smo has been the most successful target for developing Hh antagonists. There are currently nine novel Smo inhibitors in clinic trial $[6,7]$. Among them, vismodegib has been approved for treating in advanced unresectable BCC in 2012 [8].
BBR (chemical structure shown in Fig. 1a), a natural isoquinoline alkaloid, can be isolated from the rhizome, roots and stem barks of various important medicinal plants, the Berberis species. BBR exhibits multiple pharmacological activities, such as antimicrobial, antidiabetic, cardioprotective effects [9]. Additionally, it has been shown that BBR may inhibit the growth of a variety of human cancer cell lines, including prostate [4, 10], colon cancer [11], lung cancer [12, 13], nasopharyngeal cancer [14], breast cancer $[15,16]$, and leukemia cells [17]. However, the molecular mechanisms underlying the anticancer effect of BBR remain far from being fully elucidated. In this study, we identified that BBR may selectively inhibit the Hh signaling pathway activity by targeting Smo and consequently the Hh-dependent cancer growth, thus improving our knowledge of the molecular

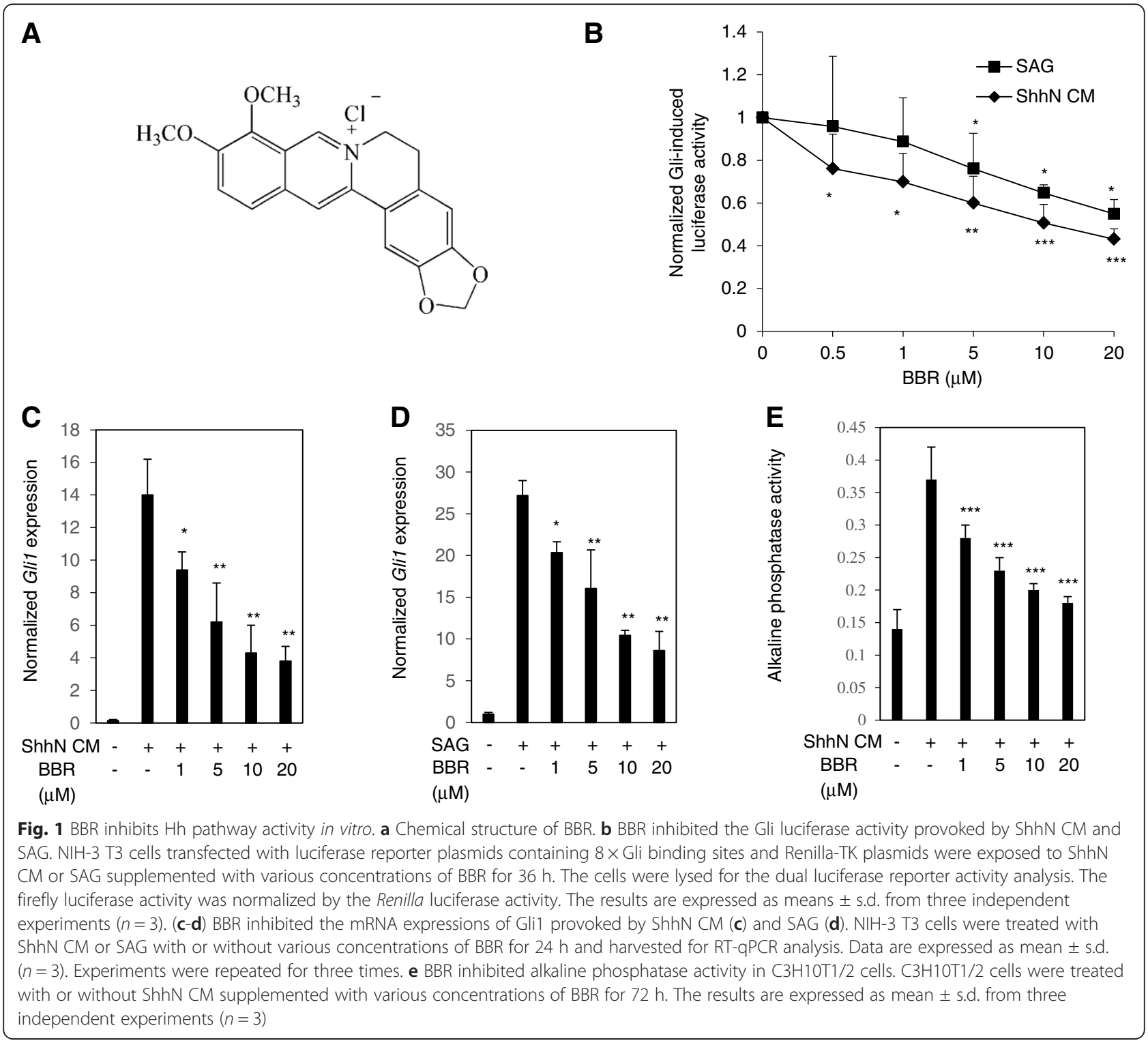


mechanisms responsible for the anticancer action of $B B R$ and contributing to the future usage of BBR as an anticancer drugs.

\section{Methods}

\section{Cell lines and culture}

The NIH-3T3 and C3H/10T1/2 mouse embryo fibroblast cells, HEK-293T human epithelial kidney cells, and LS174T colon cancer cells were obtained from the American Type Culture Collection (Manassas, VA). All these cells were routinely cultured according to the manufacturer's instructions.

The variant containing the N-terminal signaling domain of the Shh (Shh) conditioned medium (CM) were prepared as previously described [18]. Briefly, $293 \mathrm{~T}$ cells $\left(5 \times 10^{6}\right)$ were seeded in $10-\mathrm{cm}$ dishes. The plasmid harboring the ShhN were transfected into $293 \mathrm{~T}$ cells with Lipofectamine 2000 reagent (Invitrogene; Grand Island, $\mathrm{NY})$. The medium $(5 \mathrm{ml})$ in the cells were replaced with fresh medium with $0.1 \%$ serum $24 \mathrm{~h}$ post transfection. After 24, the ShhN CM were collected, and were diluted 100 -fold prior to be used for experiments.

\section{Reagents and antibodies}

BBR and PGE2 were obtained from Sigma-Aldrich (St. Louis, MO). The Hh pathway antagonists cyclopamine, GANT61, itraconazole, and BODIPY-cyclopamine were obtained from Biovision (Milpitas, CA). The Hh pathway agonist SAG was obtained from Selleck Chemicals (Houston, TX). TNF- $\alpha$, BAY $11-8072$ and H89 were purchased from Beyotime (Suzhou, China). Primary antibodies against Smo, Gli2, and Sufu and GAPDH were obtained from Santa Cruz Biotechnology (Santa Cruz, CA).

\section{Plasmids and lentivirus}

The $8 \times$ Gli1-binding site luciferase reporter $(8 \times$ GBS-luciferase) plasmid was a kind gift from Dr. Hiroshi Sasaki. The TCF/LEF-luciferase reporter plasmid, NF-кB -luciferase reporter plasmid, and TK-Renilla luciferase plasmid were purchased from Promega (Madison, WI). The Gli2 lacking the N-termianl (Gli2 $\Delta \mathrm{N})$ plasmid and ShhN plasmids were obtained from Addgene (Cambridge, MA). The Myc-DKK-tagged ORF clone of Homo sapiens Smo plasmid was purchased from Origene (Rockville, MD). The mutant human plasmid SmoM2 (W535L) was generated from wild type Smo plasmids using QuickChange Site-Directed Mutagenesis kit from Agilent (Santa Clara, CA) and was confirmed by sequencing. The Sufu-shRNA was purchased from Santa Cruz (Santa Cruz, CA).

Transient transfections were performed using Lipofectamine 2000 reagent from Invitrogen according to the manufacturer's instructions. The lentiviral stocks were prepared according to previous report [19]. Briefly, the plasmid carrying the Sufu-shRNA and three packaging plasmids were co-transfected into 293T cells using Lipofectamine 2000. The viruses were harvested $24 \mathrm{~h}$ post transfection and $4 \mathrm{ml}$ viruses were used for infected $\mathrm{NIH}-3 \mathrm{~T} 3$ cells seeded in $10-\mathrm{cm}$ dishes. Infected cells were analyzed 5-7 days post infection by western blot analyses of the expression of Sufu.

\section{Dual luciferase assays}

Cells transfected with luciferase reporter plasmids containing respective binding-sites of various transcriptional factors and Renilla-TK construct were seeded into 48well plates. After various treatments as indicated, luciferase assays were conducted using a dual luciferase assay kit according to the manufacturer's instructions (Promega) on a luminometer (Molecular Device; Sunnyvale, CA). The firefly luciferase values were normalized to Renilla values.

\section{Reverse transcription and quantitative polymerase chain reaction (RT-qPCR)}

Total RNA was extracted from cells or medullbolbatoma tissues using Trizol reagent (Takara; Dalian, China) following the manufacturer's protocol. The qPCR analyses were performed using the following primers:

\section{mGUSB: Forward: 5'-CTGCCACGGCGATGGA-3' Reverse: $5^{\prime}$-ACTGCATAATAATGGGCACTGTTG-3' mGli1: Forward: 5'-GCAGTGGGTAACATGAGTG TCT-3' \\ Reverse: 5'-AGGCACTAGAGTTGAGGAATTGT-3' mptch1: Forward: 5'-GCTACGACTATGTCTCTCA CATCAACT-3' \\ Reverse: 5'-GGCGACACTTTGATGAACCA-3'}

The mRNA levels of interested genes were normalized to those of GUSB.

\section{Western blot analysis}

NIH-3T3 cells were harvested for western blot analysis of the expression of Smo, Gli2, and Sufu according to standard procedure. The blots of GAPDH were used as loading controls.

\section{Alkaline phosphatase activity assay}

C3H10T1/2 cells were plated into 96-well plates at a density of 5000 cells per well. After treatment with or without ShhN CM supplemented with various concentrations of BBR for $72 \mathrm{~h}$. The alkaline phosphatase activity was measured using a kit from Beyotime on a plate reader (Molecular Device) at $405 \mathrm{~nm}$. 


\section{Fluorescent BODIPY-cyclopamine competition assay}

The 293T cells were seeded onto coverslips coated with poly-D-lysine in 24-well plates, followed by transfection with hSMO construct. After exposed to $1 \mathrm{uM}$ BODIPYcyclopamine supplemented with or without various compounds as indicated for $10 \mathrm{~h}$, the cells were washed with PBS, fixed with paraformaldehyde $(4 \% ; v / v)$ for 10 min, incubated with a $0.1 \%$ Triton X-100 for 15 min. The cells were then subjected to fluorescence-activated cell sorting (FACS; Becton Dickinson; San Jose, CA) analysis or were mounted with DAPI and visualized using a fluorescence microscope (Leica; Wetzlar, Germany).

\section{Medulloblastoma cells culture and Brdu assay}

Medulloblastoma cells were obtained from medulloblastoma allografts by mechanical dissociation and viable cell fractioning. The cells were maintained in Neurobasal A medium (Invitrogen) containing B-27 supplement (Invitrogen), epidermal growth factor $20 \mathrm{ng} / \mathrm{ml}$ (Invitrogen), basic fibroblastic growth factor $20 \mathrm{ng} / \mathrm{ml}$ (Invitrogen), nonessential amino acids (Invitrogen), $\mathrm{N}$-acetyl cysteine $60 \mathrm{mg} / \mathrm{ml}$, and Glutamax (Invitrogen) [20].

Medulloblastoma cells were seeded into 96-well plates, followed by treatment with various concentrations of BBR for $36 \mathrm{~h}$. The Brdu assays were conducted using the Brdu Cell Proliferation Kit (Merck Millipore; Bedford, MA) according to manufacturer's instructions.

\section{Medulloblastoma allograft model}

Primary intracranial medulloblastoma from Ptch+/-; p53-/- mouse [21, 22], which was obtained by crossing ptch+/- mice (Jackson Laboratory; Harbor, MI) with p53-/- mice (Jackson Laboratory), were harvested and allografted subcutaneously into right and left flanks of athymic nude mice (Beijing HFK Bio-Technology; Beijing, China). The well-developed tumors were harvested, cut into $1 \mathrm{~mm}^{3}$ fragments and inoculated subcutaneously into the right flank of athymic nude mice using a trocar. When the tumor volume reached 100$150 \mathrm{~mm}^{3}$, the mice were randomly assigned into control and treatment group $(n=8)$. Control group was given vehicle alone, and treatment group received BBR $(100 \mathrm{mg} / \mathrm{kg})$ via oral gavage once daily for 3 weeks. The volume of the tumors were measured twice per week using microcaliper. The tumor volume $(\mathrm{V})$ was calculated as following: $\mathrm{V}=\left[\right.$ length $\left.(\mathrm{mm}) \times \operatorname{width}^{2}\left(\mathrm{~mm}^{2}\right)\right] / 2$. The individual relative tumor volume (RTV) was calculated as following: $\mathrm{RTV}=\mathrm{Vt} / \mathrm{V} 0$, where $\mathrm{Vt}$ is the volume on each day, and V0 represents the volume at the beginning of the treatment. All animal experiments in this study were pre-approved by the Animal Care and Use Committee of the Fudan University and performed according to institutional policies.

\section{Statistical analysis}

Statistical differences were analyzed by one-way ANOVA and $P<0.05$ was considered as significant. Multiple comparison between the groups was performed using Dunnett's method. (\#p>0.05; ${ }^{*} P<0.05$, "* $P<0.01, \cdots * 0.001)$.

\section{Results}

\section{BBR inhibits $\mathrm{Hh}$ signaling in vitro}

To determine whether BBR can inhibit the Hh signaling, NIH-3T3 cells, which have good response to the Hh stimulation [23], transfected with $8 \times$ Gli1-binding site dependent firefly luciferase and Renilla Luciferase plasmids were stimulated with ShhN CM with or without various concentrations of BBR. We observed that BBR obviously inhibited the Gli-responsive reporter activity provoked by ShhN CM in a dose-dependent manner (Fig. 1b), with an IC50 value of $4.6 \pm 1.2 \mu \mathrm{M}$. Concomitantly, BBR reduced the mRNA expression of Gli1 (Fig. 1c), a transcriptional target of Gli, which served as a readout of Gli activity. Moreover, we found that BBR treatment also abolished the Gli luciferase activity (Fig. 1b) and Gli1 mRNA abundance (Fig. 1d) provoked by SAG, a small molecular compound agonist of Smo [24]. To further determine the ability of BBR of suppressing the Hh pathway activity, we conducted the alkaline phosphatase activity assay using C3H10T1/2 cells, which can express osteogenesis marker alkaline phosphatase when treated with $\mathrm{Hh}$ ligands [25, 26]. As shown in Fig. 1e, exposure of BBR obviously suppressed the alkaline phosphatase activity evoked by ShhN CM in C3H10T1/2 cells. The inhibitory effect of BBR on the alkaline phosphatase activity was not due to the nonspecific cytotoxic activity of BBR, as BBR had no effect on the cell numbers of C3H10T1/2 cells after BBR treatment for $72 \mathrm{~h}$ (data not shown). Hence, our data show that BBR may significantly inhibit the $\mathrm{Hh}$ signaling in vitro.

\section{BBR displays selectivity for inhibiting Hh pathway activity}

To rule out the possibility that BBR nonspecifically inhibits Gli luciferase activity provoked by ShhN CM, we examined the effect of BBR on other transcriptional factors, such as NF-kB, and TCF/LEF [27]. As shown in Fig. 2a-b, we observed that tumor necrosis factor $\alpha$ (TNF- $\alpha$ ) and Prostaglandin E2 (PGE2) obviously provoked the NF- $\kappa B$, and TCF/LEF luciferase activity, while BBR exhibited no inhibitory activity against either NF$\kappa B$, or TCF/LEF luciferase activity stimulated by TNF- $\alpha$ and PGE2. The BAY 11-7082, and H89 were used as positive controls for inhibition of NF- $\mathrm{BB}$, and TCF/LEF luciferase activity, respectively (Fig. 2a-b). We have demonstrated that PGE2 may activate the Gli activity in a noncanonical manner (data to be published). We then 

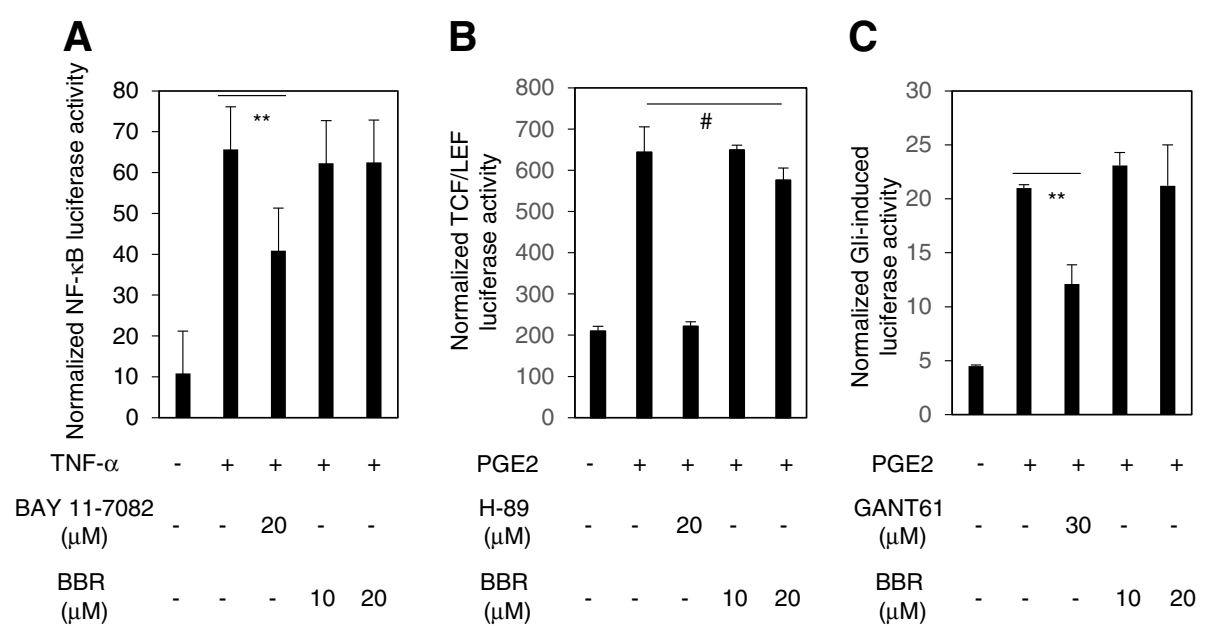

Fig. 2 BBR displays selectivity for inhibiting Hh pathway activity. a The 293 T cells transfected with luciferase reporter activity containing NF-kB binding sites and Renilla-TK plasmids were exposed to TNF-a with BAY 11-872 or various concentrations of BBR for $6 \mathrm{~h}$, and the cells were harvested for dual luciferase reporter activity analysis. The data are expressed as mean \pm s.d., $(n=3)$ from three independent experiments. $\mathbf{b}$ The LS174T cells transfected with luciferase reporter constructs containing TCF/LEF binding sites and the Renilla-TK plasmids were exposed to PGE2 with various concentrations of BBR or H89 for $24 \mathrm{~h}$, and the cells were collected for dual luciferase reporter activity analysis. The results are expressed as means \pm s.d. from three independent experiments $(n=3)$. c The LS174T cells transfected with luciferase reporter constructs containing Gli binding sites and the Renilla-TK plasmids were exposed to PGE2 with various concentrations of BBR or GANT61 for $24 \mathrm{~h}$, and the cells were collected for dual luciferase reporter activity analysis. Data are expressed as means \pm s.d. from three independent experiments $(n=3)$. ${ }^{*} p<0.05 ; \# p>0.05$

asked whether BBR may suppress the Gli activity provoked by PGE2. Interestingly, BBR failed to inhibit the Gli luciferase activity activated by PGE2 (Fig. 2c). However, the GANT61, a small molecule inhibitor targeting Gli [28], abundantly reduced the Gli luciferase activity stimulated by PGE2 (Fig. 2c). Hence, our data demonstrate that BBR exhibits unique property for suppressing Hh signaling pathway activity.

\section{BBR inhibits the Hh signaling pathway activity by targeting Smo}

Having demonstrated that BBR may specifically inhibit the Hh signaling pathway activity, we then set out to determine the molecular target of BBR for inhibiting Hh pathway activity. We first tested whether BBR may suppress the Gli luciferase activity provoked by ectopic expression of Gli. As shown in Fig. 3a, overexpression of Gli2 $\Delta \mathrm{N}$ (Fig. 3a, upper panel), a Gli2 variant missing $328 \mathrm{~N}$-terminal amino acids, in the NIH-3T3 cells obviously stimulated the Gli-luciferase activity. However, BBR had no inhibitory effect on the Gli luciferase activity provoked by Gli $2 \Delta \mathrm{N}$, while the Gli small molecular compound inhibitor GANT61 (Fig. 3a), which was used as a positive control, significantly suppressed the Gli luciferase activity. Thus, these observations ruled out the possibility that BBR inhibited the Hh signaling pathway activity by targeting Gli. Moreover, BBR also failed to affect the Gli-luciferase activity provoked by limiting the expression of Sufu (Fig. 3b), a negative regulator of Hh signaling pathway [28], suggesting that BBR inhibited the Hh pathway activity by acting upstream of Sufu. Based on these findings, we asked whether BBR inhibited the Hh pathway activity by targeting Smo, which is the most successful molecular target for developing anticancer drugs in Hh pathway. To this end, we examined the effect of BBR on the Gli-luciferase activity stimulated by ectopic expression of Smo in NIH-3T3 cells $\mathrm{N}$ (Fig. 3c, upper panel). BBR obviously inhibited the Gliluciferase activity stimulated by ectopic expression of Smo (Fig. 3c), with the IC50 value $(5.1 \pm 3.4 \mu \mathrm{M})$ similar to that for inhibiting the Gli-luciferase activity stimulated by ShhN CM $(4.6 \pm 1.2 \mu \mathrm{M})$. However, BBR exhibited little effect on the Gli-luciferase activity stimulated by SmoM2 (Fig. 3d), a frequent mutant found in cancers [29]. These data suggest that BBR inhibits the Hh pathway activity potentially through targeting Smo.

Furthermore, as shown in Fig. 1b, d, we saw that BBR also significantly inhibited the Gli-luciferase activity (Fig. 1b) and Gli1 mRNA expression (Fig. 1d) provoked by SAG, a small molecular compound agonist of Smo [24]. Interestingly, the IC50 value of BBR for inhibiting the Gli-luciferase activity provoked by ShhN CM (4.6 \pm $1.2 \mu \mathrm{M}$.) is lower than that for inhibiting Gli-luciferase activity provoked by SAG $(8.7 \pm 1.2 \mu \mathrm{M})$. Given that SAG and cyclopamine bind to the same site on Smo [24], these data suggest that BBR functions as a competitive inhibitor with SAG and most likely share the same binding site on Smo with cyclopamine. To further 


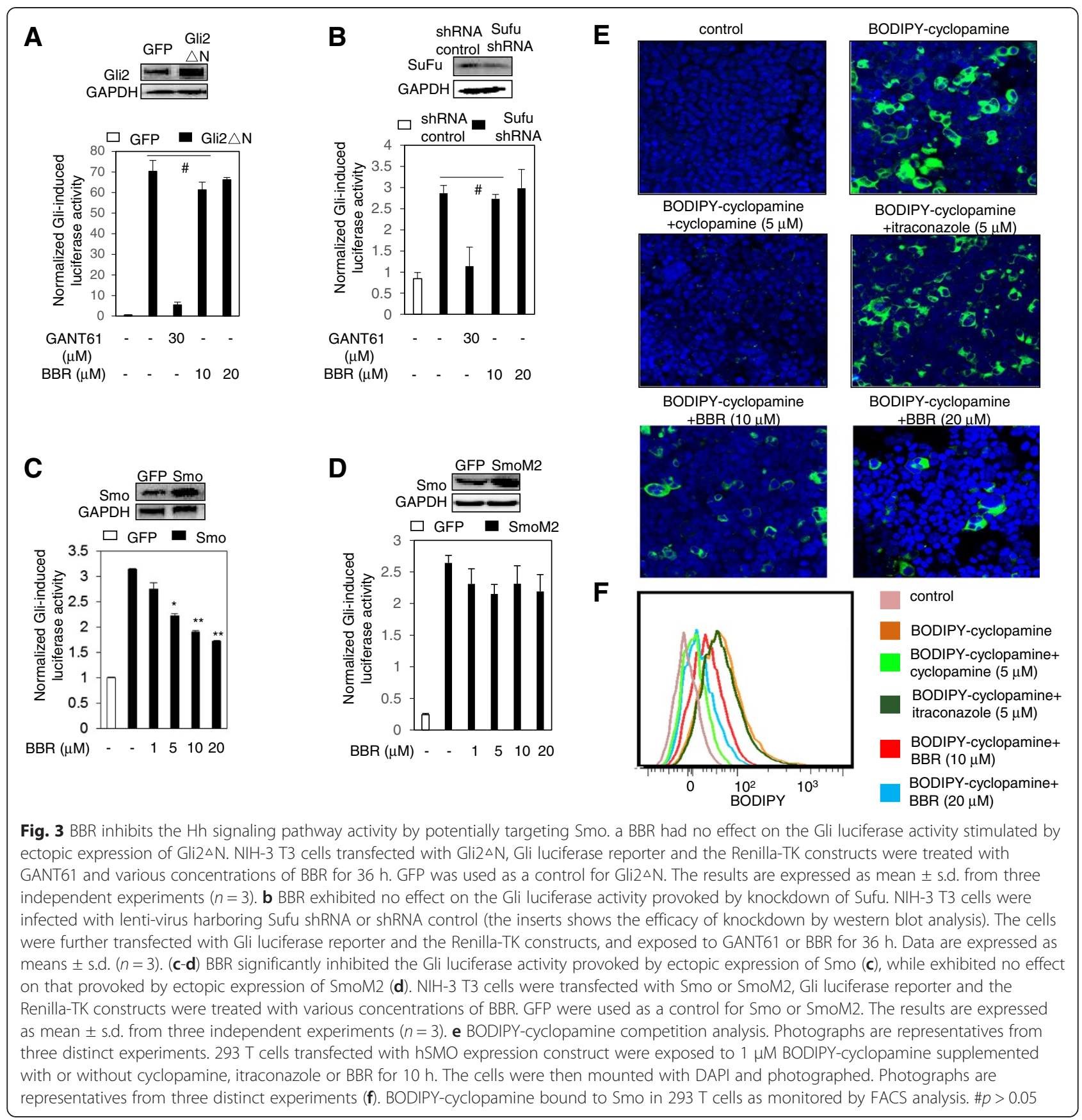

strengthen this argument, we used the BODIPYcyclopamine, a fluorescent cyclopamine derivative, assay. We observed that the binding of BODIPYcyclopamine to Smo was significantly suppressed by BBR, similar to cyclopamine, which herein served as a positive control (Fig. 3e). However, itraconazole, a Smo inhibitor binding to Smo on a distinct binding site from that of cyclopamine [20], failed to affect the binding of BODIPY-cyclopamine to Smo (Fig. 3e). These observations were further confirmed by FACS analysis
(Fig. 3f). Taken together, our data demonstrate that BBR acts on Smo to inhibit the Hh pathway.

\section{BBR inhibits the Hh-dependent medulloblastoma cell growth in vitro}

Having characterized the Hh pathway inhibitory activity of BBR, we examined the effect of BBR on the growth of Hh-dependent medulloblastoma cells isolated from medulloblastoma in ptch+/-;p53-/- mice [21, 22]. Brdu assay revealed that $\mathrm{BBR}$ dose-dependently inhibited the 

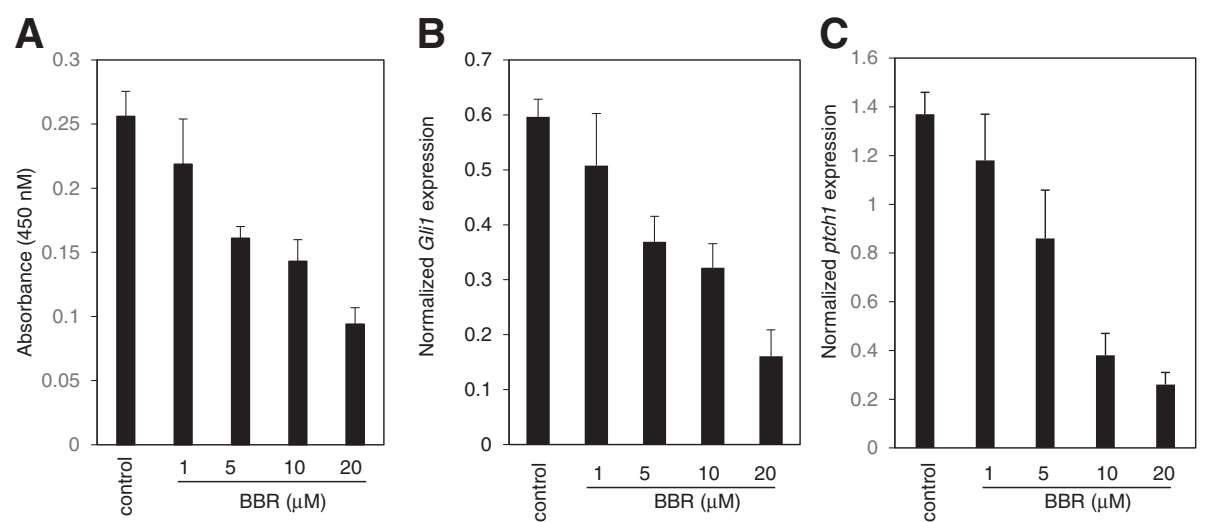

Fig. 4 BBR suppresses the proliferation of medulloblastoma cells in vitro. a BBR suppressed the proliferation of medulloblastoma cells analyzed by Brdu assay. Medulloblastoma cells were treated with various concentrations of BBR for $36 \mathrm{~h}$. Data are expressed as means \pm s.d. from three independent experiments $(n=3)$. (b-c) BBR suppressed the Gli1 mRNA (b) and ptch1 mRNA (c) expression in medulloblastoma cells. Medulloblastoma cells were exposed to various concentrations of BBR for $24 \mathrm{~h}$, and the cells were collected for RT-qPCR analysis. The results are expressed as mean \pm s.d. from three independent experiments $(n=3)$

growth of medulloblastoma cell growth (Fig. 4a), concomitantly with comparable reduction of the mRNA expression of Gli1, and ptch1 (Fig. 4b, c), which served as a readout of the Hh pathway activity. Hence, our data show that BBR may inhibit the Hh-dependent medulloblastoma cells growth in vitro through inhibiting the $\mathrm{Hh}$ pathway activity.

\section{BBR inhibits the Hh-dependent medulloblastoma growth in vivo}

To further demonstrate that BBR may inhibit the growth of the Hh-dependent medulloblastoma growth, we allografted the medulloblastoma isolated from ptch+/-;p53 -/ - mice into the Nude mice. BBR was administrated by gavage by $100 \mathrm{mg} / \mathrm{kg}$ daily. Consistent with the in vitro data, BBR significantly inhibited the medulloblastoma growth (Fig. 5a), which is accompanied with similar reduction of the mRNA expression of Gli1 (Fig. 5b), and ptch1 (Fig. 5c). Hence, our in vivo data further demonstrate that BBR may inhibit the growth of Hh-dependent medulloblastoma growth by inhibiting the Hh pathway activity.

\section{Discussion}

BBR has strong anti-inflammatory and antimicrobial activities. Owing to its excellent safety characteristics, BBR has been used as a non-prescription drug to treat diarrhea and gastroenteritis in China since 1950s. Accumulating reports show that BBR also possesses anti-cancer effects, being able to suppress the proliferation of cancer cells [9]. Previous studies have revealed various potential molecular targets responsible for the anticancer actions of BBR, such as DNA topoisomerase [30], HIF-1 $\alpha$ [31], wnt signaling pathway [11]. In this study, we demonstrate
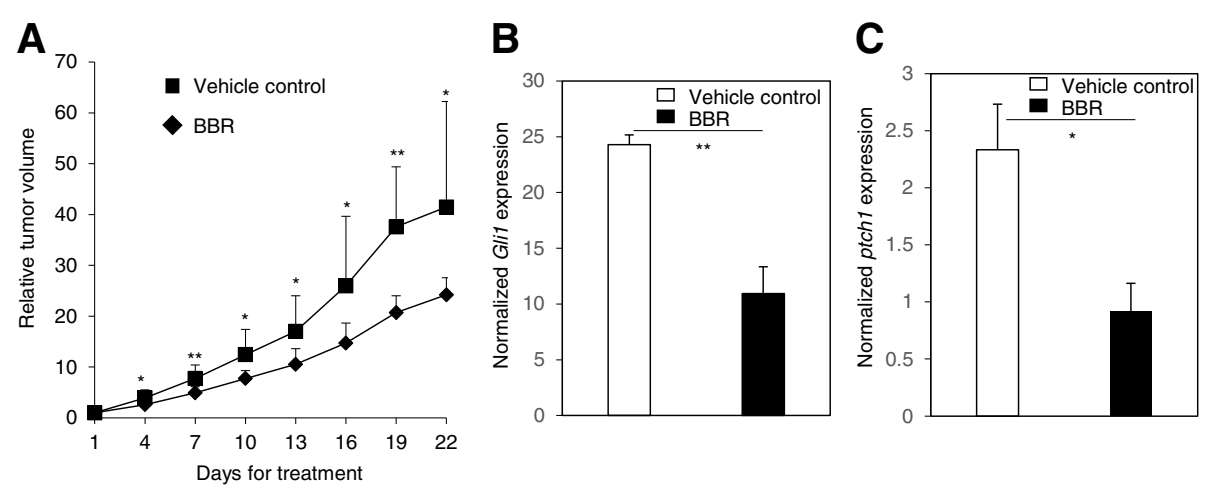

Fig. 5 BBR inhibits the growth of medullboblastoma in vivo. a Inhibitory effect of BBR on the growth of medulloblastoma in vivo. Nude mice allografted with medulloblastoma were administered the BBR $100 \mathrm{mg} / \mathrm{kg}$ by daily gavage for 21 days. RTV for indicated days was shown as mean \pm s.d. for each group of mice. (b-c) BBR inhibited the Gli1 mRNA (b) and ptch1 mRNA (c) expression in the medulloblastoma tissues isolated from nude mice. Four hours after last dose of BBR administration, the medulloblastom tissues were harvested for RT-qPCR analysis. Data are expressed as mean \pm s.d. ${ }^{*} p<0.05$ 
that BBR may selectively suppress the Hh signaling pathway activity by potentially targeting the critical component Smo, and consequently inhibit the Hh-dependent cancer growth. Therefore, our study improve our knowledge about the underlying molecular mechanisms behind the anticancer actions of BBR, thus contributing to the future usage of BBR for treating cancers driven by aberrant $\mathrm{Hh}$ pathway activity.

This study shows that BBR displays specificity when inhibiting Hh pathway activity, as reflected by no inhibitory effects of BBR on the NF-kB, TCF/LEF and Gli acitivity in response to TNF- $\alpha$ and PGE2. However, other labs have shown that BBR may suppress the NF-kB activity which consequently induces the apoptosis of multiple myeloma cells or protects against neuronal damage via suppression of glia-mediated inflammation in traumatic brain injury [32, 33]. This discrepancy between our observation and those from other labs [32,33] may be due to the distinct concentrations of BBR used. The maximum concentration used in the present study was $20 \mu \mathrm{M}$, while the concentrations which had inhibitory effect on the NF- $\mathrm{kB}$ activity in the studies from other labs ranged from 50 to $100 \mu \mathrm{M}[32,33]$.

Addiction of tumors to Hh signaling pathway for growth and metastasis has been verified with various $\mathrm{Hh}$ pathway inhibitors. The natural teratogenic compound cyclopamine, the first identified Hh pathway inhibitor, blocks Hh pathway by directly binding to Smo, slowing down tumor growth in animal models [34]. However, concerns were raised on its anticancer efficacy due to the off-target potential [35]. Moreover, the limited potency and poor oral solubility hinders its clinical development. Other more potent and selective Smo inhibitors with diverse chemical structures have been developed and are being investigated in clinical trials in a large range of advanced and metastatic cancers, such as vismodegib, sonidegib, BMS-833923, PF04449913 and LY2940680 [7]. Vismodegib has been approved for treatment of advanced BCC in 2012 [8], therefore underpinning the Smo as a molecular target for treating cancers. Among all these Smo inhibitors, the majority share the same binding site in Smo with cyclopamine, with the exceptions of itraconazole and several other antagonists newly discovered by Tao et al. [36]. In the present study, we identified that BBR can inhibit the Hh pathway activity by targeting Smo. We further identified that BBR may most likely bind Smo at the same pocket with cyclopamine, as reflected by competition of BBR for binding to Smo with SAG and BODIPY-cyclopamine. Of course, we cannot exclude the possibility that Smo acts on Smo indirectly via another molecule, as lacking direct binding assay. Hence, further verification of this argument by direct binding assay will help us to understand the characteristics of the BBR action on Smo.

\section{Conclusion}

In summary, this study shows that BBR may selectively inhibit the Hh pathway activity functioning as a Smo inhibitor potentially most likely by binding the same site in Smo with cyclopamine. Using the ptch+/-;p53-/medulloblastoma model, we also demonstrate that BBR significantly inhibit the Hh-dependent tumor growth by inhibiting Hh pathway activity. Given that BBR has been used as a non-prescription drug in China for decades, and it has a good safety profile, it should be possible for us to quickly investigate its clinical efficacy on patients with tumors dependent on the Hh pathway by either alone or in combination with other treatment strategies.

\section{Abbreviations}

ANOVA: Analysis of variance; BBR: Berberine; BCC: Basal cellular carcinoma; Brdu: 5-bromo-2-deoxyuridine; CM: Conditioned medium; Dhh: Desert hedgehog; FACS: Fluorescence-activated cell sorting; Gli2 $\triangle N$ : Gli2 lacking the N-termianl; Hh: Hedgehog; Ihh: Indian hedgehog; PGE2: Prostaglandin E2;

PTCH1: Patched1; RT-qPCR: Reverse transcription and quantitative polymerase chain reaction; RTV: Relative tumor volume; Shh: Sonic hedgehog; ShhN: The variant containing the N-terminal signaling domain of the Shh; Smo: Smoothened; SuFu: Suppressor of fused; TNF-a: Tumor necrosis factor-a.

\section{Competing interest}

The authors declare that they have no competing interests.

\section{Authors' contribution}

Participated in research design: WT, ND; conducted experiments: JW, YP, YL, and JY; wrote or contributed to the writing of the manuscript: WT, JW. All authors read and approved the final manuscript.

\section{Acknowledgements}

This study was financially supported by the National Natural Science Foundation of China (81573452), Shanghai Municipal Science \& Technology Pillar Program for Bio-pharmaceuticals (14431900400), and by State Key Laboratory of Drug Research (SIMM1501KF-09; SIMM1403KF-08).

\section{Author details}

${ }^{1}$ Department of Pharmacology, School of Pharmacy, Fudan University, 826 Zhangheng Road, Shanghai 201203, P.R. China. ${ }^{2}$ Department of Medicinal Chemistry, School of Pharmacy, Fudan University, 826 Zhangheng Rd, Shanghai 201203, P.R. China. ${ }^{3}$ State key Laboratory of Drug Research, Shanghai Institute of Materia Medica, Chinese Academy of Sciences, Shanghai 201203, China.

Received: 5 February 2015 Accepted: 10 August 2015 Published online: 22 August 2015

\section{References}

1. Ingham PW, McMahon AP. Hedgehog signaling in animal development: paradigms and principles. Genes Dev. 2001;15(23):3059-87.

2. Briscoe J, Therond PP. The mechanisms of Hedgehog signalling and its roles in development and disease. Nat Rev Mol Cell Biol. 2013;14(7):416-29.

3. Varjosalo M, Taipale J. Hedgehog: functions and mechanisms. Genes Dev. 2008:22(18):2454-72.

4. Hui CC, Angers S. Gli proteins in development and disease. Annu Rev Cell Dev Biol. 2011;27:513-37.

5. Scales SJ, de Sauvage FJ. Mechanisms of Hedgehog pathway activation in cancer and implications for therapy. Trends Pharmacol Sci. 2009;30(6):303-12.

6. Ruat M, Hoch L, Faure H, Rognan D. Targeting of Smoothened for therapeutic gain. Trends Pharmacol Sci. 2014;35(5):237-46.

7. Amakye $D$, Jagani Z, Dorsch M. Unraveling the therapeutic potential of the Hedgehog pathway in cancer. Nat Med. 2013;19(11):1410-22. 
8. Guha M. Hedgehog inhibitor gets landmark skin cancer approval, but questions remain for wider potential. Nat Rev Drug Discov. 2012;11(4):257-8.

9. Tillhon M, Guaman Ortiz LM, Lombardi P, Scovassi Al. Berberine: new perspectives for old remedies. Biochem Pharmacol. 2012;84(10):1260-7.

10. Mantena SK, Sharma SD, Katiyar SK. Berberine, a natural product, induces G1-phase cell cycle arrest and caspase-3-dependent apoptosis in human prostate carcinoma cells. Mol Cancer Ther. 2006;5(2):296-308.

11. Zhang J, Cao H, Zhang B, Xu X, Ruan H, Yi T, et al. Berberine potently attenuates intestinal polyps growth in ApcMin mice and familial adenomatous polyposis patients through inhibition of Wnt signalling. J Cell Mol Med. 2013;17(11):1484-93.

12. Katiyar SK, Meeran SM, Katiyar N, Akhtar S. p53 Cooperates berberineinduced growth inhibition and apoptosis of non-small cell human lung cancer cells in vitro and tumor xenograft growth in vivo. Mol Carcinog. 2009;48(1):24-37.

13. James MA, Fu H, Liu Y, Chen DR, You M. Dietary administration of berberine or Phellodendron amurense extract inhibits cell cycle progression and lung tumorigenesis. Mol Carcinog. 2011;50(1):1-7.

14. Tang F, Wang D, Duan C, Huang D, Wu Y, Chen $Y$, et al. Berberine inhibits metastasis of nasopharyngeal carcinoma 5-8F cells by targeting Rho kinase-mediated Ezrin phosphorylation at threonine 567. J Biol Chem. 2009:284(40):27456-66.

15. Chappell WH, Abrams SL, Franklin RA, LaHair MM, Montalto G, Cervello M, et al. Ectopic NGAL expression can alter sensitivity of breast cancer cells to EGFR, BCl-2, CaM-K inhibitors and the plant natural product berberine. Cell Cycle. 2012;11(23):4447-61.

16. Jabbarzadeh Kaboli P, Rahmat A, Ismail P, Ling KH. Targets and mechanisms of berberine, a natural drug with potential to treat cancer with special focus on breast cancer. Eur J Pharmacol. 2014;740C:584-95.

17. Zhang X, Gu L, Li J, Shah N, He J, Yang L, et al. Degradation of MDM2 by the interaction between berberine and DAXX leads to potent apoptosis in MDM2-overexpressing cancer cells. Cancer Res. 2010;70(23):9895-904.

18. Maity T, Fuse N, Beachy PA. Molecular mechanisms of Sonic hedgehog mutant effects in holoprosencephaly. Proc Natl Acad Sci U S A. 2005;102(47):17026-31.

19. Zhan X, Wang J, Liu Y, Peng Y, Tan W. GPCR-like signaling mediated by smoothened contributes to acquired chemoresistance through activating Gli. Mol Cancer. 2014;13:4.

20. Kim J, Aftab BT, Tang JY, Kim D, Lee AH, Rezaee M, et al. Itraconazole and arsenic trioxide inhibit Hedgehog pathway activation and tumor growth associated with acquired resistance to smoothened antagonists. Cancer Cell. 2013;23(1):23-34

21. Wetmore C, Eberhart DE, Curran T. Loss of p53 but not ARF accelerates medulloblastoma in mice heterozygous for patched. Cancer Res. 2001;61(2):513-6.

22. Romer JT, Kimura H, Magdaleno S, Sasai K, Fuller C, Baines $H$, et al. Suppression of the Shh pathway using a small molecule inhibitor eliminates medulloblastoma in Ptc1(+/-)p53(-/-) mice. Cancer Cell. 2004;6(3):229-40.

23. Taipale J, Chen JK, Cooper MK, Wang B, Mann RK, Milenkovic L, et al. Effects of oncogenic mutations in Smoothened and Patched can be reversed by cyclopamine. Nature. 2000;406(6799):1005-9.

24. Chen JK, Taipale J, Young KE, Maiti T, Beachy PA. Small molecule modulation of Smoothened activity. Proc Natl Acad Sci U S A 2002;99(22):14071-6.

25. Nakamura T, Aikawa T, Iwamoto-Enomoto M, Iwamoto M, Higuchi Y, Pacifici $\mathrm{M}$, et al. Induction of osteogenic differentiation by hedgehog proteins. Biochem Biophys Res Commun. 1997;237(2):465-9.

26. Williams KP, Rayhorn P, Chi-Rosso G, Garber EA, Strauch KL, Horan GS, et al. Functional antagonists of sonic hedgehog reveal the importance of the $\mathrm{N}$ terminus for activity. J Cell Sci. 1999;112(Pt 23):4405-14.

27. Shao J, Jung C, Liu C, Sheng H. Prostaglandin E2 Stimulates the betacatenin/T cell factor-dependent transcription in colon cancer. J Biol Chem. 2005;280(28):26565-72.

28. Lauth M, Bergstrom A, Shimokawa T, Toftgard R. Inhibition of GLI-mediated transcription and tumor cell growth by small-molecule antagonists. Proc Natl Acad Sci U S A. 2007;104(20):8455-60.

29. Xie J, Murone M, Luoh SM, Ryan A, Gu Q, Zhang C, et al. Activating Smoothened mutations in sporadic basal-cell carcinoma. Nature 1998;391 (6662):90-2.
30. Krishnan P, Bastow KF. The 9-position in berberine analogs is an important determinant of DNA topoisomerase II inhibition. Anticancer Drug Des. 2000;15(4):255-64.

31. Lin S, Tsai SC, Lee CC, Wang BW, Liou JY, Shyu KG. Berberine inhibits HIF-1alpha expression via enhanced proteolysis. Mol Pharmacol. 2004;66(3):612-9.

32. Hu HY, Li KP, Wang XJ, Liu Y, Lu ZG, Dong RH, et al. Set9, NF-kappaB, and microRNA-21 mediate berberine-induced apoptosis of human multiple myeloma cells. Acta Pharmacol Sin. 2013;34(1):157-66.

33. Chen CC, Hung TH, Lee CY, Wang LF, Wu CH, Ke CH, et al. Berberine protects against neuronal damage via suppression of glia-mediated inflammation in traumatic brain injury. PLoS One. 2014;9(12): e115694.

34. Chen JK, Taipale J, Cooper MK, Beachy PA. Inhibition of Hedgehog signaling by direct binding of cyclopamine to Smoothened. Genes Dev. 2002;16(21):2743-8.

35. Yauch RL, Gould SE, Scales SJ, Tang T, Tian H, Ahn CP, et al. A paracrine requirement for hedgehog signalling in cancer. Nature. 2008;455(7211):406-10.

36. Tao H, Jin Q, Koo DI, Liao X, Englund NP, Wang Y, et al. Small molecule antagonists in distinct binding modes inhibit drug-resistant mutant of smoothened. Chem Biol. 2011;18(4):432-7.

\section{Submit your next manuscript to BioMed Central and take full advantage of:}

- Convenient online submission

- Thorough peer review

- No space constraints or color figure charges

- Immediate publication on acceptance

- Inclusion in PubMed, CAS, Scopus and Google Scholar

- Research which is freely available for redistribution 